\title{
Preface. Bifurcations and Pattern Formation in Biological Applications
}

\author{
A. Morozov ${ }^{1}$, M. Ptashnyk ${ }^{2 *}$, V. Volpert ${ }^{3}$ \\ ${ }^{1}$ Department of Mathematics, University of Leicester, LE1 7RH Leicester, UK \\ ${ }^{2}$ Department of Mathematics, University of Dundee, DD1 4HN Dundee, UK \\ ${ }^{3}$ Institut Camille Jordan, UMR 5208 CNRS, University Lyon 1, 69622 Villeurbanne, France
}

\begin{abstract}
In the preface we present a short overview of articles included in the issue "Bifurcations and pattern formation in biological applications" of the journal Mathematical Modelling of Natural Phenomena.
\end{abstract}

Keywords and phrases: pattern-formation, stochastic dynamics, continuation and bifurcation analysis, chaotic dynamics, evolutionary game models

Analysis of pattern formations in biological and physical systems is a wide and active research area. Many different approaches (e.g. continuous, discrete, hybrid discrete-continuum) and techniques (e.g. linearised stability analysis and numerical continuations) are used to model and analyse the pattern formation in biological systems. The reaction-diffusion theory for pattern formation was first proposed by A. Turing in his work in 1952 on morphogenesis [21]. In 1970s H. Meinhardt and A. Gierer developed a concept of activating and inhibiting chemicals to model and analyse the formation of patterns during the developmental processes $[10,16]$. Since then the idea of diffusion-driven instability was applied to analyse pattern formations in many different biological systems [14]. Mechano-chemical theory of pattern formation developed by J.D. Murray and G. Oster [17] proposes mechanical interactions as a possible mechanism responsible for pattern formation in biological systems.

Following first analytical and numerical results, many different aspects of pattern formation in biological systems are currently being considered.

It was identified that the growth of the spatial domain of interactions plays an important role in the formation of spatial patterns $[7,12]$. In this issue the pattern formation for reaction-diffusion systems defined on evolving domains is presented by A. Madzvamuse and A.H. Chung [13]. A coupling between bulk and surface dynamics induces an interesting and important aspect of the analysis. For linearised stability analysis Lagrangian transformation of the model equations to the equations defined on a fixed domain is performed. The impact of cell death on the pattern formation in a growing bacterial biofilms described by a moving boundary model is considered by H.A. Wallace, L. Li, and F.A. Davidson in [24]. Analysis indicates that the pattern formation in biofilms depends on the height (thickness) of the biofilms.

Directed movement of cells or populations is also considered as one of the mechanisms of pattern formation, e.g. pattern formation in tumour-invasion model [6] or in the chemotaxis system [18]. The

\footnotetext{
${ }^{*}$ Corresponding author. E-mail: m.ptashnyk@dundee.ac.uk
} 
theory of spatial evolutionary game is used in the paper by A.E.F. Burgess, P.G. Schofield, S.F. Hubbard, M.A.J. Chaplain, and T. Lorenzi [5] to analyse the hybrid discrete-continuum model which includes random motion and chemotaxis. Numerical simulations of the model show that diffusion through space and chemotactic movement (i.e. up semiochemical gradients), can bring about self-generated patterns which create favourable conditions for the coexistence of two populations in situations in which the two strategies cannot coexist either in spatially homogeneous models or in cellular automaton models. The modelling framework presented in this paper potentially covers a wide range of applications including ecology (e.g. predator-prey systems), epidemiology (e.g. the invasion of new host tissues by a pathogen) and oncology (e.g. the colonisation of a new niche by tumour cells following metastasis).

The changes from regular patterns to chaos constitute an important and interesting aspect of the temporal and spatio-temporal dynamics of biological systems $[8,18]$. Complex behaviour and chaotic dynamics in the system of five ordinary differential equations modelling a tumour-immune-virus system are studied by R. Eftimie, C.K. Macnamara, J. Dushoff, J.L. Bramson and D.J.D. Earn in [9].

Analysis of mathematical models for ecosystems constitutes a vital part of the mathematical analysis of pattern formation in biological systems and is important due to limited opportunities for empirical studies of large ecosystems. In [19] applying mumerical bifurcation methods J.A. Sherratt analyses the pattern formation in the models for self-organisation observed in mussel beds and calculates the parameter region in which patterns exist and the sub-region in which these patterns are stable. He also shows how this results can be used to explain numerical observations of history-dependent wavelength selection as parameters are varied slowly. Non-local interactions can be important drivers in patterns formation and this is currently a quickly developing area of research $[1,3]$. In their work, M. Banerjee and L. Zhang explore patterns in a prey-predator model with non-local spatial interactions [2]. It is observed that the nonlocal interaction term in the prey growth has a stabilising effect on the dynamics of solutions of the model equations and can enhance the existence of stationary solutions for a wide range of parameter values.

To better understand noise induced phenomena observed in biological systems it is important to analyse the influence of noise on the dynamics of populations and on pattern formation. Chemical Master Equations [4], Stochastic ODEs and Stochastic PDEs [11] are used to describe the evolution of biological systems with external noise or intrinsic stochasticity. The influence of noise on the dynamics of an ecosystem is considered in the work by D. Valenti, A. Giuffrida, G. Denaro, N. Pizzolato, L. Curcio, B. Spagnolo, S. Mazzola, G. Basilone and A. Bonanno, presented in this issue in [22]. For Lotka-Volterra equations with a multiplicative noise the authors identify noise induced phenomena such as stochastic resonance, noise delayed extinction, and noise-induced pattern formation. The perturbation with multiplicative white noise of a deterministic model is considered by I. Siekmann and H. Malchow to model stochastic environmental fluctuations [20]. The authors show that different properties of the stochastic perturbations may not only lead to opposite predictions of survival and extinction but also considerably affect the patterns of the spatio-temporal dynamics. In [23] D. Valenti, G. Denaro, F. Giarratana, A. Giuffrida, S. Mazzola, G. Basilone, S. Aronica, A. Bonanno and B. Spagnolo consider a system of stochastic ODEs to model the dynamics of two bacterial populations responsible for food spoilage. The fluctuations are modelled by multiplicative white Gaussian noise. It is observed that the numerical solutions of the stochastic model are in a better agreement with the experimental results than the numerical solutions of the corresponding deterministic model.

\section{References}

[1] O. Aydogmus Patterns and transitions to instability in an intraspecific competition model with nonlocal diffusion and interaction, Math. Model. Nat. Phenom, 10 (6) (2015), 17-29.

[2] M. Banerjee, L. Zhang, Stabilizing role of nonlocal interaction on spatial pattern formation, Math. Model. Nat. Phenom., 115 (2016), 103-118.

[3] A. Bayliss, V.A. Volpert Patterns for competing populations with species specific nonlocal coupling Math. Model. Nat. Phenom, 10 (6) (2015), 30-47.

[4] T. Biancalani, D. Fanelli, F. Di Patti, Stochastic Turing patterns in the Brusselator model., Phys. Rev. E 81 (2010) 046215 . 
[5] A.E.F. Burgess, P.G. Schofield, S.F. Hubbard, M.A.J. Chaplain, T. Lorenzi, Dynamical patterns of coexisting strategies in a hybrid discrete-continuum spatial evolutionary game model, Math. Model. Nat. Phenom., 115 (2016), 49-64.

[6] M.A.J. Chaplain, G. Lolas, Mathematical modelling of cancer invasion of tissue: dynamic heterogeneity, Netw. Heterog. Media 1, (2006), 399-439.

[7] E.J. Crampin, E.A. Gaffney, P.K. Maini, Reaction and diffusion on growing domains: scenarios for robust pattern formation, Bull. Math. Biol. 61 (1999), 1093-1120.

[8] H. Deng, A.V. Holden, L.F. Olsen (Editors), Chaos in Biological Systems, Springer 1987.

[9] R. Eftimie, C.K. Macnamara, J. Dushoff, J.L. Bramson, D.J.D. Earn, Bifurcations and chaotic dynamics in a tumourimmune-virus system, Math. Model. Nat. Phenom., 115 (2016), 65-85.

[10] A. Gierer, H. Meinhardt, Theory of biological pattern formation, Kybernetik, 12 (1972), 30-39.

[11] J. Kelkel, C. Surulescu, On a stochastic reaction-diffusion system modeling pattern formation on seashells, J. Math. Biol., 60 (2010), 765-96.

[12] S. Kondo, R. Asal, A reaction-diffusion wave on skin of the marine angelfish Pomacanthus, Nature, 376 (1995), $765-768$.

[13] A. Madzvamuse, A.H. Chung, Analysis and simulations of coupled bulk-surface reaction-diffusion systems on exponentially evolving volumes, Math. Model. Nat. Phenom., 115 (2016), 4-32.

[14] P.K. Maini, T.E. Woolley, R.E. Baker, E.A. Gaffney, S.S. Lee, Turing's model for biological pattern formation and the robustness problem, Interface Focus, 2, (2012), 487-496.

[15] A.J. McKane, T. Biancalani, T. Rogers, Stochastic pattern formation and spontaneous polarisation: the linear noise approximation and beyond, Bull. Math. Biol. 76 (2014), 895-921.

[16] H. Meinhardt, Modelling of biological pattern formation, Academic Press, London, 1982.

[17] J.D. Murray, G. Oster, Generation of biological pattern and form, IMA J. Math. Med. Biol., 1 (1984), 1-25.

[18] K.J. Painter, T. Hillen, Spatio-temporal chaos in a chemotaxis model, Physica D, 240, (2011), 363-375.

[19] J.A. Sherratt, Using numerical bifurcation analysis to study pattern formation in mussel beds, Math. Model. Nat. Phenom., 115 (2016), 86-102.

[20] I. Siekmann, H. Malchow, Fighting enemies and noise: Competition of residents and invaders in a stochastically fluctuating environment, Math. Model. Nat. Phenom., 115 (2016), 137-157.

[21] A.M. Turing, Chemical basis of morphogenesis, Phil. Trans. R. Soc. Lond., B 237, (1952), 37-72.

[22] D. Valenti, A. Giuffrida, G. Denaro, N. Pizzolato, L. Curcio, B. Spagnolo, S. Mazzola, G. Basilone, A. Bonanno, Noise induced phenomena in population dynamics, Math. Model. Nat. Phenom., 115 (2016), 158-174.

[23] D. Valenti, G. Denaro, F. Giarratana, A. Giuffrida, S. Mazzola, G. Basilone, S. Aronica, A. Bonanno, B. Spagnolo, Modeling of sensory characteristics based on the growth of food spoilage bacteria, Math. Model. Nat. Phenom., 115 (2016), 119-136.

[24] H.A. Wallace, L. Li, F.A. Davidson, Instability in a moving boundary: heterogeneous growth of bacterial biofilms, Math. Model. Nat. Phenom., 115 (2016), 33-48. 